\title{
Collocation Method for Nonlinear Volterra-Fredholm Integral Equations
}

\author{
Jafar Ahmadi Shali ${ }^{1}$, Parviz Darania ${ }^{2}$, Ali Asgar Jodayree Akbarfam ${ }^{1}$ \\ ${ }^{1}$ Department of Mathematics and Computer Science, University of Tabriz, Tabriz, Iran \\ ${ }^{2}$ Department of Mathematics, Faculty of Science, Urmia University, Urmia, Iran \\ Email: \{j_ahmadishali,pdarania\}@tabrizu.ac.ir, Akbarfam@yahoo.com
}

Received April 18, 2012; revised May 14, 2012; accepted May 25, 2012

\begin{abstract}
A fully discrete version of a piecewise polynomial collocation method based on new collocation points, is constructed to solve nonlinear Volterra-Fredholm integral equations. In this paper, we obtain existence and uniqueness results and analyze the convergence properties of the collocation method when used to approximate smooth solutions of VolterraFredholm integral equations.
\end{abstract}

Keywords: Collocation Method; Nonlinear Volterra-Fredholm Integral Equations; Convergence Analysis; Chelyshkov Polynomials

\section{Introduction}

We shall consider the nonlinear Volterra-Fredholm integral equation

$$
y(t)=g(t)+\lambda_{1}(v y)(t)+\lambda_{2}(F y)(t), t \in I=[0, T] .
$$

The Volterra integral operators given by

$$
\begin{aligned}
& v: C(I) \rightarrow C(I) \\
& (v y)(t)=\int_{0}^{t} k_{1}(t, s, y(s)) \mathrm{d} s,
\end{aligned}
$$

where $k_{1} \in C(D \times \mathbb{R})$ and $D=\{(t, s): 0 \leq s \leq t \leq T\}$ and Fredholm integral operators given by

$$
\begin{aligned}
& F: C(I) \rightarrow C(I) \\
& (F y)(t)=\int_{0}^{T} k_{2}(t, s, y(s)) \mathrm{d} s,
\end{aligned}
$$

where $\lambda_{r}, r=1,2$ denotes (real or complex) parameters and $k_{2} \in C(I \times I \times \mathbb{R})$ and let $g \in C(I)$ be a given function.

The mentioned equations are characterized by the presence of a linear functional argument and play an important role in explaining many different phenomena.

In particular, they turn out to be fundamental when ordinary differential equations based model fail. These equations arise in industrial applications and in studies based on biology, economy, control and electro-dynamic.

Collocation method is a widely popular numerical technique in solving integral equations, differential equations, etc. When collocation method is used to solve complicated engineering problems, it has several disadvantages, that is, low efficiency, ill-conditioned, etc. Thus, different types of techniques were proposed to improve the computational performance of collocation method.

Recently, Chelyshkov has introduced sequences of polynomials in [1], which are orthogonal over the interval $[0,1]$ with the weight function 1 . These polynomials are explicitly defined by

$$
P_{m k}(t)=\sum_{j=0}^{m-k}(-1)^{j}\left(\begin{array}{c}
m-k \\
j
\end{array}\right)\left(\begin{array}{l}
m+k+1+j \\
m-k
\end{array}\right) t^{k+j}, k=0,1, \cdots, m .
$$

The polynomials $P_{m k}(t)$ have properties, which are analogous to the properties of the classical orthogonal polynomials. These polynomials can also be connected to a fixed set of Jacobi polynomials $P_{m}^{(\alpha, \beta)}(t)$. Precisely

$$
P_{m k}(t)=(-1)^{m-k} t^{k} P_{n-k}^{(0,2 k+1)}(2 t-1) .
$$

Investigating more on (4), we deduce that in the family of orthogonal polynomials $\left\{P_{m k}(t)\right\}_{k=0}^{m}$ have $k$ multiple zeros $t=0$ and $m-k$ distinct real zeros in the interval $[0,1]$. Hence, for every $m$ the polynomial $P_{m 0}(t)$ has exactly $m$ simple roots in $[0,1]$. Following [1], it can be shown that the sequence of polynomials $\left\{P_{m 0}(t)\right\}_{m=0}^{\infty}$ generate a family of orthogonal polynomials on $[0,1]$ which possesses all the properties of other classic orthogonal polynomials e.g. Legendre or Chebyshev polynomials. Therefore, if the roots of $P_{m 0}(t)$ are chosen as collocation points, then we can obtain an accurate numerical quadrature.

In the present paper, we further develop the works carried out in [2-6]. 
We discuss existence and uniqueness results and analyze the convergence properties of the collocation method when used to approximate smooth solutions of linear Volterra-Fredholm integral equations and finally, some numerical results are presented in the final section, which support the theoretical results obtained in this paper.

\section{Existence and Uniqueness Results}

Let $C(I)$ denote the Banach space continuous realvalued functions, such that $g \in C(I)$ with

$$
\|g\|_{\infty}=\max _{t \in I}|g| \text {. }
$$

Lemma 2.1. Assume $H$ is a nonempty closed set in a Banach space $V$, and that $T: H \rightarrow H$ is continuous. Suppose $T^{m}$ is a contraction for some positive integer $m$. Then, $T$ has a unique fixed-point in $H$.

Proof: For proof see [7].

Here, in integral Equation (1), we assume that $k_{i}$ for some constants $M_{i}$, satisfies a Lipschitz condition with respect to its third argument

$$
\begin{array}{r}
\left|k_{i}\left(t, s, y_{1}\right)-k_{i}\left(t, s, y_{2}\right)\right| \leq M_{i}\left|y_{1}-y_{2}\right|, \\
0 \leq s \leq t \leq T, \quad y_{i} \in \mathbb{R}, i=1,2 .
\end{array}
$$

Theorem 2.2. Assume $g$ and $k_{i}$ satisfy the condition (6) and given functions $g, k_{i}$, are continuous on their domains. Moreover, assume

$$
M_{2}<T^{-1} \text {. }
$$

Then the integral Equation (1) has a unique solution $y(t) \in C(I)$.

Proof: We define the nonlinear integral operator

$$
\begin{aligned}
& T: C(I) \rightarrow C(I), \\
& (T y)(t)=g(t)+\lambda_{1}(v y)(t)+\lambda_{2}(F y)(t), \\
& \quad t \in I=[0, T]
\end{aligned}
$$

Let us show that for $m$ sufficiently large, the operator $T^{m}$ is a contraction on $C(I)$. For $y_{1}, y_{2} \in C(I)$

$$
\begin{aligned}
& T y_{1}(t)-T y_{2}(t) \\
= & \int_{0}^{t}\left[k_{1}\left(t, s, y_{1}(s)\right)-k_{1}\left(t, s, y_{2}(s)\right)\right] \mathrm{d} s \\
+ & \int_{0}^{T}\left[k_{2}\left(t, s, y_{1}(s)\right)-k_{2}\left(t, s, y_{2}(s)\right)\right] \mathrm{d} s
\end{aligned}
$$

Then

$$
\begin{aligned}
\left|T y_{1}(t)-T y_{2}(t)\right| \leq & M_{1} \int_{0}^{t}\left|y_{1}(s)-y_{2}(s)\right| \mathrm{d} s \\
& +M_{2} \int_{0}^{T}\left|y_{1}(s)-y_{2}(s)\right| \mathrm{d} s .(10) \\
\leq & {\left[M_{1} t+M_{2} T\right]\left\|y_{1}-y_{2}\right\|_{\infty} . }
\end{aligned}
$$

Since

$$
\begin{aligned}
& T^{2} y_{1}(t)-T^{2} y_{2}(t) \\
= & \int_{0}^{t}\left[k_{1}\left(t, s, T y_{1}(s)\right)-k_{1}\left(t, s, T y_{2}(s)\right)\right] \mathrm{d} s \\
& +\int_{0}^{T}\left[k_{2}\left(t, s, T y_{1}(s)\right)-k_{2}\left(t, s, T y_{2}(s)\right)\right] \mathrm{d} s,
\end{aligned}
$$

we get

$$
\left|T^{2} y_{1}(t)-T^{2} y_{2}(t)\right| \leq\left[\frac{\left(M_{1} t\right)^{2}}{2 !}+\left(M_{2} T\right)^{2}\right]\left\|y_{1}-y_{2}\right\|_{\infty}
$$

By a mathematical induction, we obtain

$$
\begin{aligned}
& \left|T^{m} y_{1}(t)-T^{m} y_{2}(t)\right| \\
\leq & {\left[\frac{\left(M_{1} t\right)^{m}}{m !}+\left(M_{2} T\right)^{m}\right]\left\|y_{1}-y_{2}\right\|_{\infty} . }
\end{aligned}
$$

Thus

$$
\begin{aligned}
& \left\|T^{m} y_{1}(t)-T^{m} y_{2}(t)\right\| \\
\leq & {\left[\frac{\left(T M_{1}\right)^{m}}{m !}+\left(T M_{2}\right)^{m}\right]\left\|y_{1}-y_{2}\right\|_{\infty} . }
\end{aligned}
$$

Since, $0<T M_{2}<1$ then

$$
\lim _{m \rightarrow \infty}\left(T M_{2}\right)^{m}=0
$$

and

$$
\lim _{m \rightarrow \infty} \frac{M_{1}^{m}}{m !}=0,
$$

the operator $T^{m}$ is a contraction on $C(I)$ when $m$ is chosen sufficiently large. By the Lemma 2.1, the operator $T$ has a unique fixed-point in $C(I)$.

\section{Collocation Method}

Let $t_{n}=n h,\left(n=0, \cdots, N-1, t_{N}=T\right)$ define a uniform partition for $I=[0, T]$, and let

$$
\begin{aligned}
& \Pi_{N}:=\left\{0=t_{0}<t_{1}<\cdots<t_{N}=T\right\}, \\
& \sigma_{n}:=\left(t_{n}, t_{n+1}\right](0 \leq n \leq N-1) .
\end{aligned}
$$

The mesh $\Pi_{N}$ is constrained in the following sense:

$$
h=\frac{T}{N}
$$

with a given mesh $\Pi_{N}$ we associate the set of its interior points, $Z_{N}:=\left\{t_{n}: n=1, \cdots, N-1\right\}$. For a fixed $N \geq 1$ and, for given integers $d \geq-1$ and $m \geq 1$, the piecewise polynomial space $S_{m+d}^{(d)}\left(Z_{N}\right)$ is defined by

$$
S_{m+d}^{(d)}\left(Z_{N}\right):=\left\{u: C^{d}(I) \rightarrow \mathbb{R} ;\left.u\right|_{\sigma_{n}} \in \pi_{m+d}, 0 \leq n \leq N-1\right\},
$$

where $\pi_{m+d}$ denotes the set of (real) polynomials of a degree not exceeding $m+d$. The dimension of this space is given by $\operatorname{dim} S_{m+d}^{(d)}\left(Z_{N}\right)=N_{m}+d+1$. 
For integral equation, we have $d=-1$, hence, the collocation space will be $S_{m-1}^{(-1)}\left(Z_{N}\right)$. Let

$$
\left.u\right|_{\sigma_{n}} \in S_{m-1}^{(-1)}\left(Z_{N}\right)
$$

for all $t \in \sigma_{n}$ we have

$$
\begin{array}{r}
u(t)=u\left(t_{n}+s h\right)=\sum_{r=1}^{m} L_{r}(s) u\left(t_{n}+c_{r} h\right), \\
n=0,1, \cdots, N-1
\end{array}
$$

From (15) we see that an element $u \in S_{m-1}^{(-1)}\left(Z_{N}\right)$, is well defined when we know the coefficients

$$
\left\{u\left(t_{n}+c_{r} h\right)\right\}
$$

for all $n=0, \cdots, N-1$. In order to compute these coefficients, we consider the set of collocation parameters $\left\{c_{j}\right\}$, where $0 \leq c_{1}<\cdots<c_{m} \leq 1$, and define the set $X_{N}:=\left\{t_{n, j}\right\}_{j=1, n=0}^{m, N-1}$ of collocation points by

$$
t_{n, j}:=t_{n}+c_{j} h, \quad j=1, \cdots, m, n=0, \cdots, N-1 .
$$

The collocation solution $u \in S_{m-1}^{(-1)}\left(Z_{N}\right)$, will be determined by imposing the condition that $u$ satisfies the integral Equation (1) on the finite set $X_{N}$

$$
\begin{array}{r}
u(t)=g(t)+\lambda_{1}(v u)(t)+\lambda_{2}(F u)(t), \\
t \in X_{N},
\end{array}
$$

Thus, for $t=t_{n, j}=t_{n}+c_{j} h$ the collocation Equation (16) assumes the form

$$
\begin{aligned}
u\left(t_{n, j}\right)= & g\left(t_{n, j}\right)+\lambda_{1} \int_{0}^{t_{n, j}} k_{1}\left(t_{n, j}, s, u(s)\right) \mathrm{d} s \\
& +\lambda_{2} \int_{0}^{T} k_{2}\left(t_{n, j}, s, u(s)\right) \mathrm{d} s .
\end{aligned}
$$

From this equation and after some computations, we obtain

$$
\begin{aligned}
u\left(t_{n, j}\right)= & g\left(t_{n, j}\right)+\lambda_{1} h \sum_{i=0}^{n-1} \int_{0}^{1} k_{1}\left(t_{n, j}, t_{i}+s h, u\left(t_{i}+s h\right)\right) \mathrm{d} s \\
& +\lambda_{1} h \int_{0}^{c_{j}} k_{1}\left(t_{n, j}, t_{n}+s h, u\left(t_{n}+s h\right)\right) \mathrm{d} s \\
& +\lambda_{2} \sum_{i=0}^{N-1} h \int_{0}^{1} k_{2}\left(t_{n, j}, t_{i}+s h, u\left(t_{i}+s h\right)\right) \mathrm{d} s
\end{aligned}
$$

Now, by using the local Lagrange basis functions

$$
L_{1}(s)=\prod_{r=1, r \neq l}^{m} \frac{s-c_{r}}{c_{l}-c_{r}}, \quad l=1, \cdots, m,
$$

for approximating the integral terms, we use the Lagrange interpolating polynomial to approximate $k_{1}\left(t_{n, j}, s, u(s)\right)$ and $k_{2}\left(t_{n, j}, s, u(s)\right)$, we obtain

$$
\begin{aligned}
& \tilde{u}\left(t_{n, j}\right) \\
= & g\left(t_{n, j}\right)+\lambda_{1} h \sum_{i=0}^{n-1} \sum_{l=1}^{m} k_{1}\left(t_{n, j}, t_{i}+c_{1} h, u\left(t_{i}+c_{1} h\right)\right) \int_{0}^{1} L_{1}(s) \mathrm{d} s \\
& +\lambda_{1} h \sum_{l=1}^{m} k_{1}\left(t_{n, j}, t_{n}+c_{1} h, \tilde{u}\left(t_{n}+c_{l} h\right)\right) \int_{0}^{c_{j}} L_{j, l}(s) \mathrm{d} s \\
& +\lambda_{2} h \sum_{i=0}^{N-1} \sum_{l=1}^{m} k_{2}\left(t_{n, j}, t_{i}+c_{1} h, \tilde{u}\left(t_{i}+c_{l} h\right)\right) \int_{0}^{1} L_{l}(s) \mathrm{d} s .
\end{aligned}
$$

Defining the quadrature weights

$$
w_{l}:=\int_{0}^{1} L_{l}(s) \mathrm{d} s, \quad l=1, \cdots, m,
$$

and

$$
w_{j, l}:=\int_{0}^{c_{j}} L_{j, l}(s) \mathrm{d} s, \quad l, j=1, \cdots, m,
$$

the fully discretized collocation equation corresponding to (20)-(22) is thus given by

$$
\begin{aligned}
& \tilde{u}\left(t_{n, j}\right) \\
= & g\left(t_{n, j}\right)+\lambda_{1} h \sum_{i=0}^{n-1} \sum_{l=1}^{m} w_{l} k_{1}\left(t_{n, j}, t_{i}+c_{l} h, u\left(t_{i}+c_{l} h\right)\right) \\
& +\lambda_{1} h \sum_{l=1}^{m} w_{j, l} k_{1}\left(t_{n, j}, t_{n}+c_{l} h, \tilde{u}\left(t_{n}+c_{l} h\right)\right) \\
& +\lambda_{2} h \sum_{i=0}^{N-1} \sum_{l=1}^{m} w_{l} k_{2}\left(t_{n, j}, t_{i}+c_{l} h, \tilde{u}\left(t_{i}+c_{l} h\right)\right) .
\end{aligned}
$$

Note that, $\tilde{u} \in S_{m-1}^{(-1)}\left(Z_{N}\right)$ and Equation (23) represent for each $n=0,1, \cdots, N-1$, a recursive system of $m$ nonlinear algebraic equations with the unknowns $\tilde{u}\left(t_{n, j}\right)$.

\section{Global Convergence}

Let $u \in S_{m-1}^{(-1)}\left(Z_{N}\right)$ denote the (exact) collocation solution to (1) defined by (16). In our convergence analysis we examine the linear test equation

$$
\begin{aligned}
y(t)= & g(t)+\lambda_{1} \int_{0}^{t} k_{1}(t, s) y(s) \mathrm{d} s \\
& +\lambda_{2} \int_{0}^{T} k_{2}(t, s) y(s) \mathrm{d} s, \quad t \in I=[0, T],
\end{aligned}
$$

where $k_{1} \in C(D), \quad k_{2} \in C(I \times I)$. We will assume that $\lambda_{2}^{-1}$ is not in the spectrum $\sigma(F)$ of the Fredholm integral operator $F$. A comment of the convergence results to the nonlinear Equation (1) can be found at the end of this section.

Theorem 4.1. Assume that the given function in (24) satisfy $g \in C^{m}, k_{1} \in C^{m}(D), k_{2} \in C^{m}(I \times I)$. Then for all sufficiently small $h=T / N$ the constrained mesh collocation solution $u \in S_{m-1}^{(-1)}\left(Z_{N}\right)$ to (24), for all $n=0,1, \cdots, N-1$, satisfies

$$
\|\varepsilon\|_{\infty} \leq C_{m} M_{m} h^{m},
$$


where $C_{m}$ are positive constants not depending on $h$. This estimate holds for all collocation parameters $\left\{c_{j}\right\}$ with $0 \leq c_{1}<\cdots<c_{m} \leq 1$.

Proof: In each interval $\left[t_{i}+t_{i+1}\right]$, the exact solution $y$ of (24) is $m$ times continuously differentiable. This follows from the smoothness hypotheses we have imposed on $g, k_{1}, k_{2}$ and from the expressions for $y(t)$. From this it is obvious that both the left and right limits of $y(t)$, as $t$ tends to $n h$, exist and are finite. We will prove the estimate (25) by using the Peano's Theorem to write

$$
\begin{array}{r}
y\left(t_{n}+s h\right)=\sum_{r=1}^{m} L_{r}(s) y\left(t_{n}+c_{r} h\right)+h^{m} R_{m, n}(s), \\
s \in[0,1] .
\end{array}
$$

Here, we have

$$
R_{m, n}(s):=\int_{0}^{1} K_{m}(s, z) y^{(m)}\left(t_{n}+z h\right) \mathrm{d} z,
$$

and

$$
\begin{array}{r}
K_{m}(s, z)=\frac{1}{(m-1) !}\left\{(s-z)_{+}^{m-1}-\sum_{k=1}^{m} L_{k}(s)\left(c_{k}-z\right)_{+}^{m-1}\right\}, \\
z \in[0,1]
\end{array}
$$

Thus, it follows from (15) that the collocation error $\varepsilon:=y-u$ possesses to the local representation

$$
\varepsilon\left(t_{n}+s h\right)=\sum_{r=1}^{m} L_{r}(s) \varepsilon_{n, r}+h^{m} R_{m, n}(s), \quad s \in(0,1],
$$

with $\varepsilon_{n, j}=y\left(t_{n}+c_{j} h\right)-u\left(t_{n}+c_{j} h\right)$, and it satisfies the equation

$$
\begin{aligned}
\varepsilon\left(t_{n, j}\right)= & \lambda_{1} \int_{0}^{t_{n, j}} k_{1}\left(t_{n, j}, s\right) \varepsilon(s) \mathrm{d} s \\
& +\lambda_{2} \int_{0}^{T} k_{2}\left(t_{n, j}, s\right) \varepsilon(s) \mathrm{d} s .
\end{aligned}
$$

By substituting the (29) in the (30) and after some computations, we obtain

$$
\begin{aligned}
\varepsilon_{n, j}= & \lambda_{1} h \sum_{i=0}^{n-1} \sum_{r=1}^{m}\left(\int_{0}^{1} k_{1}\left(t_{n, j}, t_{i}+s h\right) L_{r}(s) \mathrm{d} s\right) \varepsilon_{i r} \\
& +\lambda_{1} h^{m+1} \sum_{i=0}^{n-1} \int_{0}^{1} k_{1}\left(t_{n, j}, t_{i}+s h\right) R_{m, i}(s) \mathrm{d} s \\
& +\lambda_{1} h \sum_{r=1}^{m} \int_{0}^{c_{j}}\left(k_{1}\left(t_{n, j}, t_{n}+s h\right) L_{r}(s) \mathrm{d} s\right) \varepsilon_{n r} \\
& +\lambda_{1} h^{m+1} \int_{0}^{c_{j}} k_{1}\left(t_{n, j}, t_{n}+s h\right) R_{m, n}(s) \mathrm{d} s \\
& +\lambda_{2} h \sum_{i=0}^{N-1} \sum_{r=1}^{m} \int_{0}^{1}\left(k_{2}\left(t_{n, j}, t_{i}+s h\right) L_{r}(s) \mathrm{d} s\right) \varepsilon_{i r} \\
& +\lambda_{2} h^{m+1} \sum_{i=0}^{N-1} \int_{0}^{1} k_{2}\left(t_{n, j}, t_{i}+s h\right) R_{m, i}(s) \mathrm{d} s
\end{aligned}
$$

Define the matrices in $L\left(\mathbb{R}^{m}\right)$,

$$
\begin{gathered}
\mathrm{B}_{1, n}^{(i)}=\left(\begin{array}{c}
\int_{0}^{1} k_{1}\left(t_{n, j}, t_{i}+s h\right) L_{r}(s) \mathrm{d} s \\
i, j=1,2, \cdots, m
\end{array}\right), \\
0 \leq i<n \leq N-1, \\
\mathrm{~B}_{2, n}=\left(\begin{array}{c}
\int_{0}^{c_{j}} k_{1}\left(t_{n, j}, t_{n}+s h\right) L_{r}(s) \mathrm{d} s \\
r, j=1,2, \cdots, m
\end{array}\right), \\
\mathrm{B}_{3, n}^{(i)}=\left(\begin{array}{c}
\int_{0}^{1} k_{2}\left(t_{n, j}, t_{i}+s h\right) L_{r}(s) d s \\
r, j=1,2, \cdots, m \\
0 \leq i<n \leq N-1,
\end{array}\right)
\end{gathered}
$$

and the vectors in $\mathbb{R}^{m}$ by

$$
\begin{gathered}
A_{1, n}^{(i)}=\left(\int_{0}^{1} k_{1}\left(t_{n, j}, t_{i}+s h\right) R_{m, i}(s) \mathrm{d} s\right)^{T}, \\
j=1,2, \cdots, m, i<n, \\
A_{2, n}=\left(\int_{0}^{c_{j}} k_{1}\left(t_{n, j}, t_{n}+s h\right) R_{m, n}(s) \mathrm{d} s\right)^{T}, \\
j=1,2, \cdots, m, \\
A_{3, n}^{(i)}=\left(\int_{0}^{1} k_{2}\left(t_{n, j}, t_{i}+s h\right) R_{m, i}(s) \mathrm{d} s\right)^{T}, \\
j=1,2, \cdots, m, i<n, \\
\varepsilon_{n}=\left(\varepsilon_{n, 1} \varepsilon_{n, 2} \cdots \varepsilon_{n m}\right)^{T}, \quad n=0,1, \cdots, N-1,
\end{gathered}
$$

by substituting the Equations (32)-(38) in Equation (31) we obtain

$$
\begin{aligned}
\varepsilon_{n}= & \lambda_{1} h \sum_{i=0}^{n-1} \mathrm{~B}_{1, n}^{(i)} \varepsilon_{i}+\lambda_{1} h^{m+1} \sum_{i=0}^{n-1} A_{1, n}^{(i)}+\lambda_{1} h \mathrm{~B}_{2, n} \varepsilon_{n} \\
& +\lambda_{1} h^{m+1} A_{2, n}+\lambda_{2} h \sum_{i=0}^{N-1} \mathrm{~B}_{3, n}^{(i)} \varepsilon_{i}+\lambda_{2} h^{m+1} \sum_{i=0}^{N-1} A_{3, n}^{(i)},
\end{aligned}
$$

this linear algebraic system may be written more concisely as

$$
\begin{aligned}
& \varepsilon_{n}-\lambda_{1} h \mathrm{~B}_{2, n} \varepsilon_{n}-\lambda_{2} h \sum_{i=0}^{N-1} \mathrm{~B}_{3, n}^{(i)} \varepsilon_{i} \\
= & \lambda_{1} h \sum_{i=0}^{n-1} \mathrm{~B}_{1, n}^{(i)} \varepsilon_{i}+\lambda_{1} h^{m+1} \sum_{i=0}^{n-1} A_{1, n}^{(i)}+\lambda_{1} h^{m+1} A_{2, n}+\lambda_{2} h^{m+1} \sum_{i=0}^{N-1} A_{3, n}^{(i)}
\end{aligned}
$$

Now, let

$$
\rho=\left[\begin{array}{cccc}
\left(I_{m}-2 \lambda_{1} h \mathrm{~B}_{2,0}\right) & 0 & \cdots & 0 \\
0 & \left(I_{m}-2 \lambda_{1} h \mathrm{~B}_{2,1}\right) & \cdots & 0 \\
\vdots & \vdots & \ddots & \cdots \\
0 & 0 & \cdots & 0 \\
0 & 0 & \cdots\left(I_{m}-2 \lambda_{1} h \mathrm{~B}_{2, N-1}\right)
\end{array}\right]
$$




$$
\begin{gathered}
Q=\left[\begin{array}{cccc}
I_{m}-2 \lambda_{2} h \mathrm{~B}_{3,0}^{(0)} & -2 \lambda_{2} h \mathrm{~B}_{3,0}^{(1)} & \ldots & -2 \lambda_{2} h \mathrm{~B}_{3,0}^{(N-1)} \\
\vdots & \vdots & \vdots & \vdots \\
-2 \lambda_{2} h \mathrm{~B}_{3, N-1}^{(0)} & -2 \lambda_{2} h \mathrm{~B}_{3, N-1}^{(1)} & \cdots I_{m} & -2 \lambda_{2} h \mathrm{~B}_{3, N-1}^{(N-1)}
\end{array}\right] \\
\varepsilon=\left[\begin{array}{c}
\varepsilon_{0} \\
\varepsilon_{1} \\
\vdots \\
\varepsilon_{N-1}
\end{array}\right]
\end{gathered}
$$

Then we have

$$
\begin{aligned}
\rho \varepsilon+Q \varepsilon= & 2 \lambda_{1} h \sum_{i=0}^{n-1} \mathrm{~B}_{1, n}^{(i)} \varepsilon_{i}+2 \lambda_{1} h^{m+1} \sum_{i=0}^{n-1} A_{1, n}^{(i)} \\
& +2 \lambda_{1} h^{m+1} A_{2, n}+2 \lambda_{2} h^{m+1} \sum_{i=0}^{N-1} A_{3, n}^{(i)} .
\end{aligned}
$$

Since the kernel $K_{i}$ is continuous on their domains, the elements of the matrixes $\mathrm{B}_{2, n}, n=0,1, \cdots, N-1$ are all bounded. By using the Neumann Lemma the inverse of the matrix $\beta_{n}=I_{m}-2 \lambda_{1} h \mathrm{~B}_{2, n}$ exists whenever

$$
\lambda_{1} h\left\|\mathrm{~B}_{2, n}\right\|<\frac{1}{2}
$$

for some matrix norm. This clearly holds whenever $h$ is sufficiently small. In other words, there is an $\bar{h}>0$ so that for any mesh $\Pi_{N}$ with $h<\bar{h}$, each matrix $\beta_{n}$ has a uniformly bounded inverse. Therefore, matrix $\rho$ has a uniformly bounded inverse.

Also, the invertibility of the $N_{m} \times N_{m}$ block matrix $Q$ now depends not only on $h$ but also on $\lambda_{2}$ it is guaranteed if $\left|\lambda_{2}\right| \cdot\|F\|<\frac{1}{2}$, where

$$
\|F\|:=\max _{t \in I} \int_{0}^{T}\left|k_{2}(t, s)\right| \mathrm{d} s \leq \bar{k}_{2}^{*} T=\bar{k}_{2},
$$

assuming that $\left|k_{2}(t, s)\right| \leq \bar{k}_{2}^{*}$ and the elements of the matrixes $Q$ are all bounded. Thus from (44), we get

$$
\left(I_{m}+\Re\right) \varepsilon=\zeta_{n}
$$

where

$$
\Re=\rho^{-1} Q
$$

and

$$
\begin{aligned}
\zeta_{n}=\rho^{-1}\left\{2 \lambda_{1} h \sum_{i=0}^{n-1} \mathrm{~B}_{1, n}^{(i)} \varepsilon_{i}+2 \lambda_{1} h^{m+1} \sum_{i=0}^{n-1} A_{1, n}^{(i)}\right. \\
\left.+2 \lambda_{1} h^{m+1} A_{2, n}+2 \lambda_{2} h^{m+1} \sum_{i=0}^{N-1} A_{3, n}^{(i)}\right\} .
\end{aligned}
$$

It is clear that, matrix $\mathfrak{R}$ has a uniformly bounded inverse and the elements of the matrixes $\Re$ are all bounded. Note that, from these assumptions and $\mathfrak{R}^{-1}=Q^{-1} \rho$, there exists a constant $D_{0}<\infty$ so that for all mesh di- ameters $h \in(0, \bar{h})$, the uniform bound

$$
\left\|\left(I_{m}+\Re\right)^{-1}\right\|_{1} \leq P_{0},
$$

holds. Here, for $B \in L\left(\mathbb{R}^{m}\right),\|B\|_{1}$ denotes the matrix (operator) norm induced by the $l^{1}$-norm in $\mathbb{R}^{m}$. Assume that $\|\mathfrak{R}\|<1$ and $\left\|B_{1, n}^{(i)}\right\|_{1} \leq P_{1}$ for $0 \leq i<n \leq N-1$, and $\left\|\rho^{-1}\right\|_{1} \leq P_{2}$.

From (46) and (48) we have

$$
\begin{aligned}
\|\varepsilon\|_{1}= & \left\|\left(I_{m}+\Re\right)^{-1} \zeta_{n}\right\|_{1} \leq P_{0}\left\|\zeta_{n}\right\|_{1}, \\
\left\|\zeta_{n}\right\|_{1}= & \| \rho^{-1}\left\{2 \lambda_{1} h \sum_{i=0}^{n-1} \mathrm{~B}_{1, n}^{(i)} \varepsilon_{i}+2 \lambda_{1} h^{m+1} \sum_{i=0}^{n-1} A_{1, n}^{(i)}\right. \\
& \left.\left.+2 \lambda_{1} h^{m+1} A_{2, n}+2 \lambda_{2} h^{m+1} \sum_{i=0}^{N-1} A_{3, n}^{(i)}\right\}\right\} \|_{1} \\
\leq & 2 P_{0} P_{1}\left|\lambda_{1} h\right| \sum_{i=0}^{n-1}\left\|\varepsilon_{i}\right\|_{1}+2 P_{0}\left\{\left|\lambda_{1} h^{m+1}\right| n m \bar{k}_{1} M_{m} K_{m}\right. \\
& \left.+\left|\lambda_{1} h^{m+1}\right| m k_{1} M_{m} K_{m}+\left|\lambda_{2} h^{m+1}\right| N m \bar{k}_{2} M_{m} K_{m}\right\},
\end{aligned}
$$

and hence

$$
\|\varepsilon\|_{1} \leq \beta_{0} \sum_{i=0}^{n-1}\left\|\varepsilon_{i}\right\|_{1}+\beta_{1} M_{m} h^{m+1}
$$

where

$$
\begin{aligned}
& \beta_{0}=2 P_{0} P_{1}\left|\lambda_{1} h\right|, \beta_{1}=2 P_{0} m K_{m}\left[\overline{k_{1}}\left|\lambda_{1}\right|(n+1)+\left|\lambda_{2}\right| N \overline{k_{2}}\right] \\
& \left\|A_{1, n}^{(i)}\right\|_{1} \leq m \overline{k_{1}} K_{m} M_{m}, i<n,\left\|A_{2, n}\right\|_{1} \leq m \overline{k_{1}} K_{m} M_{m}, \\
& \left\|A_{3, n}^{(i)}\right\|_{1} \leq m \overline{k_{2}} K_{m} M_{m}, i<n, M_{m}:=\left\|y^{(m)}\right\|_{\infty}, \\
& k_{m}:=\max _{s \in[0,1]} \int_{0}^{1}\left|K_{m}(s, z)\right| \mathrm{d} z,\|v\|_{\infty}:=\max _{t \in I} \int_{0}^{1}\left|k_{1}(s, v)\right| \mathrm{d} v \leq \overline{k_{1}} .
\end{aligned}
$$

Also,

$$
\left\|\varepsilon_{n}\right\|_{1}=\sum_{j=1}^{m}\left|\varepsilon_{n j}\right| \leq \sum_{l=0}^{N-1}\left(\sum_{j=1}^{m}\left|\varepsilon_{l j}\right|\right)=\|\varepsilon\|_{1}
$$

Then, from (52) and (54), we have

$$
\left\|\varepsilon_{n}\right\|_{1} \leq \beta_{0} \sum_{i=0}^{n-1}\left\|\varepsilon_{i}\right\|_{1}+\beta_{1} M_{m} h^{m+1}, n=0,1, \cdots, N-1 .
$$

Now, by using the discrete Gronwall inequality, we have

$$
\left\|\varepsilon_{n}\right\|_{1} \leq \beta_{2} M_{m} h^{m+1}, n=0,1, \cdots, N-1,
$$

where $\beta_{2}=\beta_{1} \exp \left(n \beta_{0}\right)<\infty$.

Now, by using the local error representation (29) this yields, setting $W_{m}:=\max _{j}\left\|L_{j}\right\|_{\infty}$,

$$
\left|\varepsilon\left(t_{n}+s h\right)\right| \leq W_{m}\left\|\varepsilon_{n}\right\|_{1}+h^{m} K_{m} M_{m} \leq C_{m} M_{m} h^{m},
$$


uniformly for $s \in[0,1]$ and $0 \leq n \leq N-1$, where

$$
C_{m}=W_{m} \beta_{2}+K_{m} \text {. }
$$

The is equivalent to the estimate $\|\varepsilon\|_{\infty} \leq C_{m}\left\|y^{(m)}\right\|_{\infty} h^{m}$.

We conclude this section with a comment regarding the extension of the results of Theorem 1 to the nonlinear Equation (1). Under the assumption of the existence of a (unique) solution $y(t)$ on $I$, the nonlinear analogue of the error Equation (30) is

$$
\begin{aligned}
\varepsilon\left(t_{n, j}\right)= & \lambda_{1} \int_{0}^{t_{n, j}}\left[k_{1}\left(t_{n, j}, s, y(s)\right)-k_{1}\left(t_{n, j}, s, u(s)\right)\right] \mathrm{d} s \\
& +\lambda_{2} \int_{0}^{T}\left[k_{2}\left(t_{n, j}, s, y(s)\right)-k_{2}\left(t_{n, j}, s, u(s)\right)\right] \mathrm{d} s .
\end{aligned}
$$

If the partial derivatives $\frac{\partial k_{i}}{\partial y}, i=1,2$ are continuous and bounded on $D \times D_{1}$ with

$$
D_{1}:=\{y \in \mathbb{R}:|y-y(s)|<M, s \in I\},
$$

for some $M<\infty$, and if $h>0$ is sufficiently small, then (55) may again be written in the form (30). The roles of $k_{i}$ are now assumed by

$$
H_{i}(t, s):=\frac{\partial k_{i}\left(t, s, z_{i}(s)\right)}{\partial y}, i=1,2
$$

where $z_{i}(s):=\theta_{i} y(s)+\left(1-\theta_{i}\right) u(s), 0 \leq \theta_{i}(s) \leq 1$.

Hence, the above proof is easily adapted to deal with the nonlinear case (1), and so the convergence results of Theorem 1 remain valid for nonlinear Volterra-Fredholm integral equations.

\section{Presentation of Results}

In this section, we report on the numerical result of test problem solved by the proposed method of this article.

Typical forms of collocation parameters $c_{j}$ are:

Gauss points: Zeros of $P_{m}(2 t-1)$;

Radou I points: Zeros of $P_{m}(2 t-1)+P_{m-1}(2 t-1)$;

Radou II points: Zeros of $P_{m}(2 t-1)-P_{m-1}(2 t-1)$;

Chelyshkov points: Zeros of

$$
P_{m 0}(t)=(-1)^{m} P_{m}^{(0,1)}(2 t-1) ;
$$

where $P_{m}(t)$ and $P_{m}^{(\alpha, \beta)}(t)$ are Legendre and Jacobi polynomials, respectively.

Example 5.1. The nonlinear Volterra-Fredholm integral equation in $[0,1]$

$$
\begin{array}{r}
y(t)=t-t^{2}-1+2 \int_{0}^{t} y(s) \mathrm{d} s+3 \int_{0}^{1} y^{2}(s) \mathrm{d} s, \\
0 \leq s \leq t \leq 1,
\end{array}
$$

Table 1. Error for example 1.

\begin{tabular}{cccccc}
\hline$m$ & $N$ & $\frac{\text { Guass }}{\|e\|_{\infty}}$ & $\frac{\text { RadauI }}{\|e\|_{\infty}}$ & $\frac{\text { Radau II }}{\|e\|_{\infty}}$ & $\frac{\text { Chelyshkov }}{\|e\|_{\infty}}$ \\
\hline 3 & 2 & $2 \times 10^{-30}$ & $2 \times 10^{-30}$ & $2 \times 10^{-30}$ & $1 \times 10^{-30}$ \\
3 & 4 & $2 \times 10^{-30}$ & $2 \times 10^{-30}$ & $3 \times 10^{-30}$ & $1 \times 10^{-30}$ \\
3 & 8 & $2 \times 10^{-30}$ & $2 \times 10^{-30}$ & $2 \times 10^{-30}$ & $2 \times 10^{-30}$ \\
\hline
\end{tabular}

has the following analytical solution $y(t)=t$ therefore, provides an example to verify the accuracy of this method.

Table 1 shows the maximum errors involved presented method with $h=\frac{1}{2}, \frac{1}{4}, \frac{1}{8}$, along with the exact solution.

For computational purposes, in the test problem different forms of kernels are considered. All the computations were carried out with Maple. In each cases of Example the obtained nonlinear equations was solved by the Newton's method.

The result for collocation points $c_{j}$ are presented in Table 1 which indicates that the numerical solutions obtained from (56) and step sizes equal to $\frac{1}{2}, \frac{1}{4}$ and $\frac{1}{8}$ are nearly identical. These results indicate that, if we use the Chelyshkov points, then we obtain the numerical solutions of minimum error.

\section{Conclusion}

We have shown that the collocation method yields an efficient and very accurate numerical method for the approximation of solutions to Volterra-Fredholm integral equations. Also we have shown that, if the roots of $P_{m 0}(t)$ are chosen as collocation points, then we can obtain an accurate numerical quadrature.

\section{Acknowledgements}

The authors truly appreciate the comments made by referees.

\section{REFERENCES}

[1] V. S. Chelyshkov, "Alternative Orthogonal Polynomials and Quadratures," Electronic Transactions on Numerical Analysis, Vol. 25, No. 7, 2006, pp. 17-26.

[2] H. Brunner, "Collocation Methods for Volterra Integral and Related Functional Equations (Cambridge Monographs on Applied and Computational Mathematics)," Vol. 15, Cambridge University Press, Cambridge, 2004.

[3] H. Brunner, "Implicitly Linear Collocation Methods for Nonlinear Volterra Equations," Applied Numerical Mathematics, Vol. 9, No. 3-5, 1992, pp. 235-247. 
doi:10.1016/0168-9274(92)90018-9

[4] H. Brunner, "High-Order Collocation Methods for Singular Volterra Functional Equations of Neutral Type," Applied Numerical Mathematics, Vol. 57, No. 5-7, 2007, pp. 533-548. doi:10.1016/j.apnum.2006.07.006

[5] H. Brunner, "The Numerical Solution of Weakly Singular Volterra Functional Integro-Differential Equations with Variable Delays," Communications on Pure and Applied Analysis, Vol. 5, No. 2, 2006, pp. 261-276. doi:10.3934/cpaa.2006.5.261

[6] A. T. Diogo, S. McKee and T. Tang, "A Hermite-Type Collocation Method for the Solution of an Integral Equation with a Certain Weakly Singular Kernel," IMA Journal of Numerical Analysis, Vol. 11, No. 4, 1991, pp. 595605. doi:10.1093/imanum/11.4.595

[7] K. E. Atkinson and W. Han, "Theoretical Numerical Analysis," Springer, Berlin, 2009. 